\title{
THE LINEAR QUADRATIC CONTROL PROBLEM FOR INFINITE DIMENSIONAL SYSTEMS WITH UNBOUNDED INPUT AND OUTPUT OPERATORS*
}

\author{
A. J. PRITCHARD $\dagger$ AND D. SALAMON $\ddagger$
}

\begin{abstract}
This paper establishes a general semigroup framework for solving quadratic control problems with infinite dimensional state space and unbounded input and output operators.
\end{abstract}

Key words. infinite dimensional systems, linear quadratic control, unbounded inputs and outputs, semigroups

AMS(MOS) subject classification. $93 \mathrm{C} 25$

1. Introduction. The object of this paper is to present a general semigroup theoretic framework for solving the linear quadratic control problem (LQCP) for systems with an infinite dimensional state space and unbounded input and output operators.

The LQCP has been one of the central research problems in the area of mathematical systems theory for more than twenty years. This is partly due to its beautiful mathematical structure. Furthermore, the LQCP provides a link betweein the area of optimal control and structure theory for linear control systems, and last, but not least, the infinite time quadratic cost problem leads to a numerically stable procedure for stabilizing a linear system by feedback.

For finite dimensional systems the LQCP is now well understood (see e.g. Willems [28], Wonham [29]) and a more or less complete generalization of the finite dimensional theory has been developed for infinite dimensional systems with bounded input and output operators (see e.g. Datko [6], Curtain and Pritchard [4], Lions [19], Gibson [10], Bensoussan, Delfour and Mitter [2], Zabczyk [30]).

In many dynamical systems, the control and observation processes are severely limited. For example there may be delays in the control actuators and measurement devices. Also for systems described by partial differential equations (PDE) it may not be possible to influence or sense the state at each point of the spatial domain. Instead controls and sensors are restricted to a few points or parts of the boundary. Modelling such limitations results in unbounded input and output operators. For infinite dimensional systems with unbounded input and output operators the LQCP has recently been studied by various authors. One of the first papers in this direction was by Lukes and Russell [20] and involved spectral operators. The classical reference for parabolic systems is of course the book of Lions [19]. His results have only recently been generalized to parabolic systems with a larger degree of unboundedness in the input and output operators (Pollock and Pritchard [22], Balakrishnan [1], Flandoli [9], Lasiecka and Triggiani [16], Sorine [26], [27]). The LQCP for first order hyperbolic PDE's has been studied by Russell [23]. Lasiecka and Triggiani [18] consider the higher dimensional wave equation with Dirichlet boundary control. In their paper the resulting optimal feedback operator is unbounded. For retarded systems with input delays we refer to Ichikawa [12] and Delfour [8] and for neutral systems with output delays to Datko [7] and Ito and Tarn [14].

\footnotetext{
* Received by the editors February 20, 1984, and in revised form October 7, 1985.

$\dagger$ Control Theory Centre, University of Warwick, England CV4 7AL.

‡ Forschungsschwerpunkt Dynamische Systeme, Universität Bremen, West Germany.
} 
All of these papers deal with very specific classes of infinite dimensional systemsso far, no attempt has apparently been made to develop a general semigroup theoretic approach for the infinite dimensional LQCP with unbounded input and output operators which applies both to parabolic and hyperbolic PDE's as well as to retarded and neutral functional differential equations (FDE). In the present paper we fill this gap. An essential feature in our approach is that the semigroup $S(t)$ which describes the dynamics of the homogeneous equation is not assumed to have any smoothing properties. This is possible by means of the theory developed in Salamon [25, Chap. 1.3] and provides the basis for our approach to the LQCP.

In $\S 2$ we solve the finite quadratic control problem in the general semigroup theoretic framework. In particular, we derive the existence of a unique-nonnegative solution $P(t)$ of the operator differential Riccati equation and we show that the unique optimal control is given by a time-varying feedback law involving this operator $P(t)$. We point out that the solution operator $P(t)$ of the Riccati equation has smoothing properties and that the associated feedback operator is bounded.

Section 3 is devoted to the infinite time problem and the solution is described in terms of the operator algebraic Riccati equation. The solution of the algebraic Riccati equation is derived as the limit operator of the solutions of the differential equation on the interval [0,T] as $T$ tends to infinity. Generalizing the results of Zabczyk [30], we establish relationships between the stabilizability and detectability properties of the system and existence and uniqueness results for the algebraic Riccati equation.

In $\S 4$ we show how our general theory applies to parabolic and hyperbolic PDE's with boundary control as well as for neutral FDE's with output delays. For these special classes of infinite dimensional systems we do not derive substantial new results. We do, however, obtain a number of known results, which have not even been published, as simple straightforward consequences of our general theory. Another application of this theory to retarded FDE's with delays in control and observation will be the subject of a follow up paper.

2. Finite time control. In a formal sense our basic model is

$$
\begin{aligned}
& \dot{x}(t)=A x(t)+B u(t), \quad x\left(t_{0}\right)=x_{0}, \\
& y(t)=C x(t), \quad t_{0} \leqq t \leqq t_{1},
\end{aligned}
$$

where $u(\cdot) \in L^{2}\left[t_{0}, t_{1} ; U\right], y(\cdot) \in L^{2}\left[t_{0}, t_{1} ; Y\right], U$ and $Y$ are Hilbert spaces and $A$ is the infinitesimal generator of a strongly continuous semigroup $S(t)$ on a Hilbert space $H$. In order to allow for possible unboundedness of the operators $B$ and $C$, we assume that $B \in \mathscr{L}(U, V)$, and $C \in \mathscr{L}(W, Y)$ where $W, V$ are Hilbert spaces such that

$$
W \subset H \subset V
$$

with continuous dense injections. Of course, we interpret (2.1) in the mild form which means that its solution $x(t)$ is given by the variation-of-constants formula

$$
x(t)=S\left(t-t_{0}\right) x_{0}+\int_{t_{0}}^{t} S(t-\sigma) B u(\sigma) d \sigma, \quad t_{0} \leqq t \leqq t_{1} .
$$

In order to make this formula precise and to allow for trajectories in all three spaces $W, H, V$, we have to assume that $S(t)$ is also a strongly continuous semigroup on $W$ and $V$ and that the following hypotheses are satisfied.

(H1) There exists some constant $b>0$ such that $\int_{t_{0}}^{t_{1}} S\left(t_{1}-\sigma\right) B u(\sigma) d \sigma \in W$ and $\left\|\int_{t_{0}}^{t_{1}} S\left(t_{1}-\sigma\right) B u(\sigma) d \sigma\right\|_{W} \leqq b\|u(\cdot)\|_{L^{2}\left(t_{0}, t_{1} ; U\right)}$ for every $u(\cdot) \in L^{2}\left(t_{0}, t_{1} ; U\right)$. 
(H2) There exists some constant $c>0$ such that $\left\|C S\left(\cdot-t_{0}\right) x\right\|_{L^{2}\left(t_{0}, t_{1} ; Y\right)} \leqq c\|x\|_{V}$ for every $x \in W$.

Remarks 2.1. (i) Hypothesis (H1) implies that for every $x_{0} \in W$ and every $u(\cdot) \in$ $L^{2}\left(t_{0}, t_{1} ; U\right)$ formula $(2.3 \mathrm{a})$ defines a continuous function $x(\cdot)$ on the interval $\left[t_{0}, t_{1}\right]$ with values in $W$. Hence the output can in this case be defined by

$$
y(t)=C S\left(t-t_{0}\right) x_{0}+C \int_{t_{0}}^{t} S(t-\sigma) B u(\sigma) d \sigma, \quad t_{0} \leqq t \leqq t_{1},
$$

and is a continuous function on the interval $\left[t_{0}, t_{1}\right]$ with values in $Y$. If $x_{0} \in V$, then $x(\cdot)$ is only a continuous function with values in $V$ and $(2.3 \mathrm{~b})$ does not make sense directly. But if (H2) is satisfied, then for any $x_{0} \in V$ we will use the expression $C S\left(t-t_{0}\right) x_{0}, t_{0} \leqq t \leqq t_{1}$, to denote the function in $L^{2}\left(t_{0}, t_{1} ; Y\right)$ which is obtained by continuous extension to $x_{0} \in V$ of the operator which maps $x_{0} \in W$ into $C S\left(\cdot-t_{0}\right) x_{0} \in$ $L^{2}\left(t_{0}, t_{1} ; Y\right)$. In this sense the right-hand side of $(2.3 \mathrm{~b})$ is a well defined $L^{2}$-function of $t$ with values in $Y$.

(ii) In the above sense the expression $C S(t) B u$ has a well defined meaning as a function of $t$ for every $u \in U$. But the expression $C S(t-\sigma) B u(\sigma)$ will in general not be a well defined function of $\sigma$. Therefore the operator $C$ cannot be taken under the integral sign in (2.3b).

(iii) In the following we identify the Hilbert spaces $H, U, Y$ with their duals. Then it follows from (2.2) by duality that

$$
V^{*} \subset H \subset W^{*}
$$

with continuous, dense injections. Furthermore, the adjoint semigroup $S^{*}(t)$ is a strongly continuous semigroup on all three spaces $V^{*}, H, W^{*}$.

(iv) Hypothesis (H1) is satisfied if and only if the following dual statement holds.

$$
\left\|B^{*} S^{*}\left(t_{1}-\cdot\right) x\right\|_{L^{2}\left(t_{0}, t_{1} ; U\right)} \leqq b\|x\|_{W^{*}}, \quad x \in V^{*} .
$$

This is a simple consequence of the identity

$$
\left\langle x, \int_{t_{0}}^{t_{1}} S\left(t_{1}-\sigma\right) B u(\sigma) d \sigma\right\rangle_{V^{*}, V}=\int_{t_{0}}^{t_{1}}\left\langle B^{*} S^{*}\left(t_{1}-\sigma\right) x, u(\sigma)\right\rangle_{U} d \sigma
$$

for $x \in V^{*}$ and $u(\cdot) \in L^{2}\left(t_{0}, t_{1} ; U\right)$ and the fact that $W^{* *}=W$. Similarly, the dual statement of $(\mathrm{H} 2)$ is the following.

$\left(\mathrm{H} 2^{*}\right)$ For every $y(\cdot) \in L^{2}\left(t_{0}, t_{1} ; Y\right)$ we have

$$
\left\|\int_{t_{0}}^{t_{1}} S^{*}\left(\tau-t_{0}\right) C^{*} y(\tau) d \tau\right\|_{V^{*}} \leqq c\|y(\cdot)\|_{L^{2}\left(t_{0}, t_{1} ; Y\right)} .
$$

(v) In view of hypothesis $\left(\mathrm{H} 1^{*}\right)$ the expression $B^{*} S^{*}(t) x$ has a well defined meaning as an $L^{2}$ function of $t$ for every $x \in W^{*}$, in particular when $x=C^{*} y$ with $y \in Y$.

Associated with the control system (2.3) is the performance index

$$
J(u)=\left\langle x\left(t_{1}\right), G x\left(t_{1}\right)\right\rangle_{V, V^{*}}+\int_{t_{0}}^{t_{1}}\left[\|C x(t)\|_{Y}^{2}+\langle u(t), R u(t)\rangle_{U}\right] d t,
$$

where $G \in \mathscr{L}\left(V, V^{*}\right)$ is a nonnegative definite operator and $R \in \mathscr{L}(U)$ satisfies

$$
\langle u, R u\rangle_{U} \geqq \varepsilon\|u\|_{U}^{2}
$$

for some $\varepsilon>0$ and every $u \in U$.

Now let us consider system (2.3) with the feedback control

$$
u_{F}(t)=F(t) x(t), \quad t_{0} \leqq t \leqq t_{1},
$$


where $F(t) \in \mathscr{L}(V, U)$ is strongly continuous on the interval $\left[t_{0}, t_{1}\right]$. Then we may define a mild evolution operator $\Phi_{F}(t, s) \in \mathscr{L}(V), t_{0} \leqq s \leqq t \leqq t_{1}$, via

$$
\Phi_{F}(t, s) x=S(t-s) x+\int_{s}^{t} S(t-\sigma) B F(\sigma) \Phi_{F}(\sigma, s) x d \sigma
$$

(see Curtain and Pritchard [4]).

Remarks 2.2. (i) It follows from (2.6) that $\Phi_{F}(t, s)$ satisfies the equation

$$
\Phi_{F}(t, s) x-x=\int_{s}^{t} \Phi_{F}(t, \sigma)[A+B F(\sigma)] x d \sigma, \quad t_{0} \leqq s \leqq t \leqq t_{1}
$$

for every $x \in \mathscr{D}_{V}(A)$ (the domain of $A$ regarded as an unbounded, closed operator on $V)$. Equivalently the function $s \rightarrow \Phi_{F}(t, s) x \in V$ is continuously differentiable on the interval $\left[t_{0}, t\right]$ for every $x \in \mathscr{D}_{V}(A)$ and satisfies

$$
\frac{\partial \Phi_{F}(t, s) x}{\partial s}=-\Phi_{F}(t, s)[A+B F(s)] x, \quad t_{0} \leqq s \leqq t \leqq t_{1}
$$

(see Curtain and Pritchard [4].)

(ii) It is well known that the evolution operator satisfies the equation

$$
\Phi_{F}(t, s) x=S(t-s) x+\int_{s}^{t} \Phi_{F}(t, \sigma) B F(\sigma) S(\sigma-s) x d \sigma
$$

for $t_{0} \leqq s \leqq t \leqq t_{1}$ and $x \in V$. (See Curtain and Pritchard [4].)

(iii) Often we will consider the feedback system with an additional forcing input $v(\cdot)$ so that

$$
u(t)=F(t) x(t)+v(t)
$$

in (2.3). It follows easily from (2.9) that-for this control function-the corresponding solution of $(2.3)$ is given by

$$
x(t)=\Phi_{F}\left(t, t_{0}\right) x_{0}+\int_{t_{0}}^{t} \Phi_{F}(t, \sigma) B v(\sigma) d \sigma, \quad t_{0} \leqq t \leqq t_{1} .
$$

(iv) Using (2.6), it is easy to see that $\Phi_{F}(t, s)$ is also a strongly continuous evolution operator on $W$ and $V$ and has the following properties.

(H1') There exists a constant $b^{\prime}>0$ such that

$$
\left\|\int_{t_{0}}^{t} \Phi_{F}(t, \sigma) B u(\sigma) d \sigma\right\|_{W} \leqq b^{\prime}\|u(\cdot)\|_{L^{2}\left(t_{0}, t ; U\right)}
$$

for every $u(\cdot) \in L^{2}\left(t_{0}, t_{1} ; U\right)$ and every $t \in\left[t_{0}, t_{1}\right]$.

$\left(\mathrm{H} 2^{\prime}\right)$ There exists a constant $c^{\prime}>0$ such that

$$
\left\|C \Phi_{F}(\cdot, s) x\right\|_{L^{2}\left(s, t_{1} ; Y\right)} \leqq c^{\prime}\|x\|_{V}
$$

for every $x \in W$ and every $s \in\left[t_{0}, t_{1}\right]$

The dual properties are the following:

$\left(\mathrm{H} 1^{\prime}\right)^{*}$ The inequality

$$
\left\|B^{*} \Phi_{F}^{*}(t, \cdot) x\right\|_{L^{2}\left(t_{0}, t ; U\right)} \leqq b^{\prime}\|x\|_{W^{*}}
$$

holds for every $x \in V^{*}$ and every $t \in\left[t_{0}, t_{1}\right]$. 
$\left(\mathrm{H} 2^{\prime}\right)^{*}$ The inequality

$$
\left\|\int_{s}^{t_{1}} \Phi_{F}^{*}(\tau, s) C^{*} y(\tau) d \tau\right\|_{V^{*}} \leqq c^{\prime}\|y(\cdot)\|_{L^{2}\left(s, t_{1} ; Y\right)}
$$

holds for every $y(\cdot) \in L^{2}\left(t_{0}, t_{1} ; Y\right)$ and every $s \in\left[t_{0}, t_{1}\right]$.

Using the condition $\left(\mathrm{H}^{\prime}\right)$ and its dual, we can define a strongly continuous operator $P_{F}(t) \in \mathscr{L}\left(V, V^{*}\right)$, by

$$
\begin{aligned}
P_{F}(t) x= & \Phi_{F}^{*}\left(t_{1}, t\right) G \Phi_{F}\left(t_{1}, t\right) x+\int_{t}^{t_{1}} \Phi_{F}^{*}(\tau, t) C^{*} C \Phi_{F}(\tau, t) x d \tau \\
& +\int_{t}^{t_{1}} \Phi_{F}^{*}(\tau, t) F^{*}(\tau) R F(\tau) \Phi_{F}(\tau, t) x d \tau
\end{aligned}
$$

for $t_{0} \leqq t \leqq t_{1}$ and $x \in V$. Then the cost of the feedback control (2.5) corresponding to an initial state $x_{0} \in V$ is given by

$$
J\left(u_{F}\right)=\left\langle x_{0}, P_{F}\left(t_{0}\right) x_{0}\right\rangle_{V, V^{*}} .
$$

If the initial state is in $H$, then this expression can be interpreted via the inner product in $H$.

Remark 2.3. The adjoint operator of $P_{F}(t) \in \mathscr{L}\left(V, V^{*}\right)$ is still an operator from $V$ to $V^{*}$ and coincides with $P_{F}(t)$. In this sense one can say that $P_{F}(t)$ is self adjoint. Equivalently, the operator $i^{-1} P_{F}(t)$ on the Hilbert space $V$ is self adjoint with respect to the inner product on $V$ where $i: V \rightarrow V^{*}$ is the canonical isometric isomorphism. Finally, $P_{F}(t)$ is self adjoint in the above sense if and only if its restriction to $H$ is a self adjoint operator on $H$.

A formula comparing the cost of an arbitrary control $u(\cdot) \in L^{2}\left(t_{0}, t_{1} ; U\right)$ with the cost of the feedback control (2.5) will play an important role in our analysis. In the proof of this result we will need to interchange some integrals. At some points this becomes a delicate problem since we will have to operate with terms like $C \Phi_{F}(t, s) B$. In order to make the results precise, we need a third hypothesis.

(H3) Suppose that

$$
Z=\mathscr{D}_{V}(A) \subset W
$$

with a continuous, dense embedding where the Hilbert space $Z$ is endowed with the graph norm of $A$, regarded as an unbounded, closed operator on $V$. This assumption is not very restrictive. It is satisfied by all known examples of systems which satisfy $(\mathrm{H} 1)$ and $(\mathrm{H} 2)$ if the spaces $W$ and $V$ are chosen appropriately. In the following we summarize some important consequences of (H3).

Remarks 2.4. (i) If (H3) is satisfied, then $A$ can be regarded as a bounded operator from $Z$ into $V$. Correspondingly $A^{*}$ becomes a bounded operator from $V^{*}$ into $Z^{*}$. On the other hand $A$ can be restricted to a closed, densely defined operator on $Z$. Its adjoint in this sense coincides with the above operator $A^{*}: V^{*} \rightarrow Z^{*}$ (Salamon [25, Lemma 1.3.2]) and moreover

$$
\mathscr{D}_{W^{*}}\left(A^{*}\right) \subset \mathscr{D}_{Z^{*}}\left(A^{*}\right)=V^{*} .
$$

(ii) It is a well-known fact from semigroup theory that

$$
T_{t} x=\int_{0}^{t} S(s) x d s \in \mathscr{D}_{V}(A)=Z
$$


for every $x \in V$ and every $t \geqq 0$. If (H3) is satisfied, then $T_{t}$ is a strongly continuous family of bounded, linear operators from $V$ into $W$. It is easy to see that the adjoint operator $T_{t}^{*} \in \mathscr{L}\left(W^{*}, V^{*}\right)$ is given by

$$
T_{t}^{*} x=\int_{0}^{t} S^{*}(s) x d s \in \mathscr{D}_{W^{*}}\left(A^{*}\right) \subset V^{*}
$$

for $x \in W^{*}$ and $t \geqq 0$.

(iii) If (H1), (H2) and (H3) are satisfied, then the following equation holds for every $u \in U$ and every $t \geqq 0$

$$
C \int_{0}^{t} S(s) B u d s=C T_{t} B u=\int_{0}^{t} C S(s) B u d s .
$$

This seems like a trivial fact; however, we were not able to establish this identity without assuming (H3). Note that the LHS of the above equation has to be interpreted in terms of $(\mathrm{H} 1)$ and the RHS in terms of $(\mathrm{H} 2)$. For establishing the equation one must approximate $B u \in V$ by a sequence of elements in $W$. Then the term on the LHS will not converge in general unless range $T_{t} \subset W$.

LEMMA 2.5. Suppose that (H1), (H2), (H3) are satisfied; let $F(t) \in \mathscr{L}(V, U)$, $t_{0} \leqq t \leqq t_{1}$, be strongly continuous and let $\Phi_{F}(t, s) \in \mathscr{L}(V) \cap \mathscr{L}(W)$ be defined by (2.6). Moreover, let $u(\cdot) \in L^{2}\left(t_{0}, t_{1} ; U\right)$ and $y(\cdot) \in L^{2}\left(t_{0}, t_{1} ; U\right)$ be given. Then

$$
\int_{t_{0}}^{t_{1}} \int_{s}^{t_{1}}\left\langle C \Phi_{F}(t, s) B u(s), y(t)\right\rangle_{Y} d t d s=\int_{t_{0}}^{t_{1}}\left\langle C \int_{t_{0}}^{t} \Phi_{F}(t, s) B u(s) d s, y(t)\right\rangle_{Y} d t
$$

where the first expression must be interpreted in terms of (H2) and the second in terms of $(\mathrm{H} 1)$.

Proof. First note that, by (2.6) and (H1), $\Phi_{F}(t, s)-S(t-s) \in \mathscr{L}(V, W)$. Hence it is enough to establish the desired equation with $\Phi_{F}(t, s)$ replaced by $S(t-s)$. Secondly it is easy to see that with $T_{t} \in \mathscr{L}(V, W)$ defined as in Remark 2.4(ii) the equations

$$
\begin{aligned}
& x(t)=\int_{t_{0}}^{t} S(t-s) B u(s) d s=\int_{t_{0}}^{t} T_{t-s} B \dot{u}(s) d s \in W, \\
& z(s)=\int_{s}^{t_{1}} S^{*}(t-s) C^{*} y(t) d t=-\int_{s}^{t_{1}} T_{t-s}^{*} C^{*} \dot{y}(t) d t \in V^{*}
\end{aligned}
$$

hold for $u(\cdot) \in \mathscr{C}^{1}\left[t_{0}, t_{1} ; U\right]$ with $u\left(t_{0}\right)=0$ and $y(\cdot) \in \mathscr{C}^{1}\left[t_{0}, t_{1} ; Y\right]$ with $y\left(t_{1}\right)=0$. Interchanging integrals, we obtain from these identities that

$$
\begin{aligned}
\int_{t_{0}}^{t_{1}}\langle C x(t), y(t)\rangle_{Y} d t & =\int_{t_{0}}^{t}\langle B u(s), z(s)\rangle_{V, V^{*}} d s \\
& =\int_{t_{0}}^{t_{1}} \int_{s}^{t_{1}}\langle C S(t-s) B u(s), y(t)\rangle_{Y} d t d s .
\end{aligned}
$$

Now the statement of the lemma follows from the fact that both sides of this equation depend continuously on $u(\cdot) \in L^{2}\left[t_{0}, t_{1} ; U\right]$ and $y(\cdot) \in L^{2}\left[t_{0}, t_{1} ; Y\right]$.

Now we are in the position to prove the desired comparison formula for the feedback control (2.5).

LemMA 2.6. Suppose that (H1), (H2), (H3) are satisfied; let $F(t) \in \mathscr{L}(V, U)$ be strongly continuous on the interval $\left[t_{0}, t_{1}\right]$ and let $P_{F}(t) \in \mathscr{L}\left(V, V^{*}\right)$ be defined by (2.12) 
and (2.6). Then the following equation holds for every $x_{0} \in V$ and every $u(\cdot) \in L^{2}\left(t_{0}, t_{1} ; U\right)$

$$
J(u)-\left\langle x_{0}, P_{F}\left(t_{0}\right) x_{0}\right\rangle_{V, V^{*}}=\int_{t_{0}}^{t_{1}}\left\langle R^{-1} B^{*} P_{F}(t) x(t)\right.
$$

$$
\begin{gathered}
\left.\quad+u(t), R\left[R^{-1} B^{*} P_{F}(t) x(t)+u(t)\right]\right\rangle d t \\
-\int_{t_{0}}^{t_{1}}\left\langle R^{-1} B^{*} P_{F}(t) x(t)\right. \\
\left.\quad+F(t) x(t), R\left[R^{-1} B^{*} P_{F}(t) x(t)+F(t) x(t)\right]\right\rangle d t
\end{gathered}
$$

where $x(t), t_{0} \leqq t \leqq t_{1}$, is given by (2.3).

Proof. We sketch only the main steps of the proof for the case $x_{0} \in W$. Let $x(t)$ be the mild solution of (2.1) given by (2.3) and define

$$
\begin{aligned}
& v(t)=u(t)-F(t) x(t) \\
& z(t)=\int_{t_{0}}^{t} \Phi_{F}(t, s) B v(s) d s=x(t)-\Phi_{F}\left(t, t_{0}\right) x_{0}
\end{aligned}
$$

for $t_{0} \leqq t \leqq t_{1}$ (see Remark 2.2(iii)). Then applying Lemma 2.5, we can obtain

$$
\begin{aligned}
& 2 \operatorname{Re} \int_{t_{0}}^{t_{1}} \int_{s}^{t_{1}}\left\langle C \Phi_{F}(t, s) z(s), C \Phi_{F}(t, s) B v(s)\right\rangle d t d s \\
& \quad=\operatorname{Re} \int_{t_{0}}^{t_{1}} \int_{s}^{t_{1}}\left\langle C z(t), C \Phi_{F}(t, s) B v(s)\right\rangle d t d s=\int_{t_{0}}^{t_{1}}\|C z(t)\|_{Y}^{2} d t
\end{aligned}
$$

and therefore, again using Lemma 2.5,

$$
\begin{aligned}
2 \operatorname{Re} & \int_{t_{0}}^{t_{1}} \int_{s}^{t_{1}}\left\langle C \Phi_{F}(t, s) x(s), C \Phi_{F}(t, s) B v(s)\right\rangle d t d s \\
& =2 \operatorname{Re} \int_{t_{0}}^{t_{1}}\left\langle C \Phi_{F}\left(t_{1}, t_{0}\right) x_{0}, C z(t)\right\rangle d t+\int_{t_{0}}^{t_{1}}\|C z(t)\|_{Y}^{2} d t \\
& =\int_{t_{0}}^{t_{1}}\|C x(t)\|_{Y}^{2} d t-\int_{t_{0}}^{t_{1}}\left\|C \Phi_{F}\left(t, t_{0}\right) x_{0}\right\|_{Y}^{2} d t .
\end{aligned}
$$

Analogous identities can be derived in a more straightforward way when $C^{*} C$ is replaced by $G \in \mathscr{L}\left(V, V^{*}\right)$ or $F^{*}(t) R F(t) \in \mathscr{L}\left(V, V^{*}\right)$. Using first (2.12) and then these identities, we get

$$
\begin{aligned}
2 \operatorname{Re} \int_{t_{0}}^{t_{1}}\left\langle P_{F}(s) x(s), B v(s)\right\rangle d s \\
=\left\langle x\left(t_{1}\right), G x\left(t_{1}\right)\right\rangle-\left\langle\Phi_{F}\left(t_{1}, t_{0}\right) x_{0}, G \Phi_{F}\left(t_{1}, t_{0}\right) x_{0}\right\rangle \\
\quad+\int_{t_{0}}^{t_{1}}\|C x(t)\|^{2} d t-\int_{t_{0}}^{t_{1}}\left\|C \Phi_{F}\left(t, t_{0}\right) x_{0}\right\|^{2} d t \\
\quad+\int_{t_{0}}^{t_{1}}\langle F(t) x(t), R F(t) x(t)\rangle d t \\
\quad-\int_{t_{0}}^{t_{1}}\left\langle F(t) \Phi_{F}\left(t, t_{0}\right) x_{0}, R F(t) \Phi_{F}\left(t, t_{0}\right) x_{0}\right\rangle d t \\
=J(u)-\left\langle x_{0}, P_{F}\left(t_{0}\right) x_{0}\right\rangle-\int_{t_{0}}^{t_{1}}\langle u(t), R u(t)\rangle d t
\end{aligned}
$$




$$
+\int_{t_{0}}^{t_{1}}\langle F(t) x(t), R F(t) x(t)\rangle d t .
$$

It is easy to see that this equation implies (2.15).

We are now able to prove the main result of this section.

TheOREM 2.7. Let (H1), ( $\mathrm{H} 2)$ and $(\mathrm{H} 3)$ be satisfied. Then there exists a unique strongly continuous self adjoint, nonnegative operator $P(t) \in \mathscr{L}\left(V, V^{*}\right) t_{0} \leqq t \leqq t_{1}$, solving the integral Riccati equation.

$$
\begin{aligned}
P(t) x= & \Phi^{*}\left(t_{1}, t\right) G \Phi\left(t_{1}, t\right) x \\
& +\int_{t}^{t_{1}} \Phi^{*}(s, t)\left[C^{*} C+P(s) B R^{-1} B^{*} P(s)\right] \Phi(s, t) x d s
\end{aligned}
$$

for $x \in W$ and $t_{0} \leqq t \leqq t_{1}$ where $\Phi(s, t)=\Phi_{F}(s, t)$ is the evolution operator defined by (2.6) with $F(t)=-R^{-1} B^{*} P(t) \in \mathscr{L}(V, U)$. Furthermore there is a unique optimal control which minimizes the performance index (2.4) subject to (2.3). This optimal control is given by the feedback control law

$$
u_{F}(t)=-R^{-1} B^{*} P(t) x(t)
$$

and the optimal cost is

$$
J\left(u_{F}\right)=\left\langle x_{0}, P\left(t_{0}\right) x_{0}\right\rangle .
$$

Proof. We regard (2.16) as a fixed point problem which is to be solved by iteration. Let us define the sequence $P_{k}(t) \in \mathscr{L}\left(V, V^{*}\right)$ recursively through

$$
P_{0}(t)=0, \quad P_{k}(t)=P_{F}(t), \quad F(t)=-R^{-1} B^{*} P_{k-1}(t)
$$

for $k \in \mathbb{N}$ and $t_{0} \leqq t \leqq t_{1}$, where $P_{F}(t)$ is given by (2.12). Let us also define

$$
\Phi_{k}(s, t)=\Phi_{F}(s, t), \quad F(t)=-R^{-1} B^{*} P_{k}(t),
$$

so that

$$
\begin{aligned}
\left\langle z, P_{k+1}(t) x\right\rangle_{V, V^{*}}= & \left\langle\Phi_{k}\left(t_{1}, t\right) z, G \Phi_{k}\left(t_{1}, t\right) x\right\rangle_{V, V^{*}} \\
& +\int_{t}^{t_{1}}\left\langle C \Phi_{k}(s, t) z, C \Phi_{k}(s, t) x\right\rangle_{Y} d s \\
& +\int_{t}^{t_{1}}\left\langle B^{*} P_{k}(s) \Phi_{k}(s, t) z, R B^{*} P_{k}(s) \Phi_{k}(s, t) x\right\rangle_{U} d s
\end{aligned}
$$

holds for $t_{0} \leqq t \leqq t_{1}$ and $x \in W$. Applying Lemma 2.6 to $F(t)=-R^{-1} B^{*} P_{k-1}(t)$ and $u_{k}(t)=-R^{-1} B^{*} P_{k}(t) x(t)$, we obtain

$\left\langle x_{0}, P_{k+1}\left(t_{0}\right) x_{0}\right\rangle=J\left(u_{k}\right)=\left\langle x_{0}, P_{k}\left(t_{0}\right) x_{0}\right\rangle$

$$
\begin{gathered}
-\int_{t_{0}}^{t_{1}}\left\langle\left[P_{k}(\tau)-P_{k-1}(\tau)\right] x(\tau), B R^{-1} B^{*}\right. \\
\left.\qquad\left[P_{K}(\tau)-P_{k-1}(\tau)\right] x(\tau)\right\rangle d \tau \\
\leqq\left\langle x_{0}, P_{k}\left(t_{0}\right) x_{0}\right\rangle
\end{gathered}
$$

for $k \in \mathbb{N}$ and $x_{0} \in V$. Thus the sequence $\left\langle x_{0}, P_{k}\left(t_{0}\right) x_{0}\right\rangle_{V, V^{*}}, k \in \mathbb{N}$, is monotonically decreasing and positive. Applying Kato's result [15, p. 454, Thm. 3.3] to the monotonically decreasing sequence of nonnegative operators $i^{-1} P_{k}\left(t_{0}\right)$ on $V$ where $i: V \rightarrow V^{*}$ is 
the canonical isomorphism (Remark 2.3), we obtain the strong convergence of this sequence to a nonnegative limit operator on $V$. Hence the operators $P_{k}\left(t_{0}\right) \in \mathscr{L}\left(V, V^{*}\right)$ converge strongly to a nonnegative self adjoint operator $P\left(t_{0}\right) \in \mathscr{L}\left(V, V^{*}\right)$. The same conclusion is valid for every $t \in\left[t_{0}, t_{1}\right]$ since $t_{0} \leqq t_{1}$ can be chosen arbitrarily.

Moreover, (2.20) shows that the operators $P_{k}(t) \in \mathscr{L}\left(V, V^{*}\right), t_{0} \leqq t \leqq t_{1}, k \in \mathbb{N}$ are uniformly bounded. Hence the limit operator $P(t) \in \mathscr{L}\left(V, V^{*}\right)$ is strongly measurable and uniformly bounded on the interval $\left[t_{0}, t_{1}\right]$. Therefore we can introduce a strongly continuous evolution operator $\Phi(s, t)=\Phi_{F}(s, t) \in \mathscr{L}(V) \cap \mathscr{L}(W)$ which is defined by (2.6) with $F(t)=-R^{-1} B^{*} P(t)$.

Our next step is to show that the function $\Phi_{k}(\cdot, t) x-\Phi(\cdot, t) x \in \mathscr{C}\left[t, t_{1} ; W\right]$ converges to zero in the sup-norm for every $x \in V$ and every $t \in\left[t_{0}, t_{1}\right]$. For this purpose let us consider the identity

$$
\begin{aligned}
\Phi(s, t) x-\Phi_{k}(s, t) x= & \int_{t}^{s} S(s-\tau) B R^{-1} B^{*}\left[P_{k}(\tau)-P(\tau)\right] \Phi(\tau, t) x d \tau \\
& -\int_{t}^{s} S(s-\tau) B R^{-1} B^{*} P_{k}(\tau)\left[\Phi(\tau, t) x-\Phi_{k}(\tau, t) x\right] d \tau
\end{aligned}
$$

and apply Gronwall's lemma. Then the desired convergence of $\Phi_{k}(s, t) x$ follows from the pointwise strong convergence of $P_{k}(\tau)$ to $P(\tau)$ together with the dominated convergence theorem.

As a consequence of this convergence result we obtain that $\Phi_{k}(s, t)$ converges to $\Phi(s, t)$ both in $\mathscr{L}(V)$ and in $\mathscr{L}(W)$ and that this convergence is uniform for $t \leqq s \leqq t_{1}$ ( $t$ fixed). This allows us to apply the dominated convergence theorem to formula (2.19) and hence $P(t)$ satisfies the integral Riccati equation (2.16). Finally it follows easily from (2.16) together with the strong continuity of $\Phi(s, t)$ and $\Phi^{*}(s, t)$ in both variables and in both spaces $V$ and $W$ that the operator $P(t) \in \mathscr{L}\left(V, V^{*}\right)$ is strongly continuous on the interval $\left[t_{0}, t_{1}\right]$. Thus we have proved the existence of a solution to (2.16).

In order to prove the uniqueness for the solution of $(2.16)$ together with the statements on the optimal control, let us assume that $P(t) \in \mathscr{L}\left(V, V^{*}\right)$ is any strongly continuous, nonnegative solution of (2.16). Moreover, let $x_{0} \in V, u(\cdot) \in L^{2}\left(t_{0}, t_{1} ; U\right)$ be given, let $x(t) \in V$ be the corresponding solution of (2.1) which is given by (2.3) and define $v(t)=u(t)+R^{-1} B^{*} P(t) x(t)$ for $t_{0} \leqq t \leqq t_{1}$. Then it follows from Lemma 2.6 that

$$
J(u)=\left\langle x_{0}, P\left(t_{0}\right) x_{0}\right\rangle+\int_{t_{0}}^{t_{1}}\langle v(t), R v(t)\rangle d t .
$$

Hence the optimal control is unique and given by the feedback law (2.17) and the optimal cost is given by (2.18). Moreover, we conclude from (2.21) that $\left\langle x_{0}, P\left(t_{0}\right) x_{0}\right\rangle=$ $\left\langle x_{0}, \hat{P}\left(t_{0}\right) x_{0}\right\rangle$ for any two nonnegative solutions $P(t), \hat{P}(t) \in \mathscr{L}\left(V, V^{*}\right)$ of $(2.16)$ and any $x_{0} \in V$. Since $t_{0} \leqq t_{1}$ can be chosen arbitrarily, this proves the uniqueness of the solution to $(2.16)$.

The following result shows that the integral Riccati equation (2.16) can be converted into a differential Riccati equation.

Proposition 2.8. Suppose that (H1), (H2) and (H3) are satisfied and let $P(t) \in$ $\mathscr{L}\left(V, V^{*}\right)$ be a nonnegative, self adjoint, strongly continuous operator on the interval $\left[t_{0}, t_{1}\right]$. Moreover, let the evolution operator $\Phi(s, t)=\Phi_{F}(s, t) \in \mathscr{L}(V)$ be defined by (2.6) with $F(t)=-R^{-1} B^{*} P(t)$. Then the following statements are equivalent.

(i) Equation (2.16) holds for every $x \in W$ and every $t \in\left[t_{0}, t_{1}\right]$. 
(ii) For every $x \in W$ and every $t \in\left[t_{0}, t_{1}\right]$ the following equation holds

$$
P(t) x=\Phi^{*}\left(t_{1}, t\right) G S\left(t_{1}-t\right) x+\int_{t}^{t_{1}} \Phi^{*}(s, t) C^{*} C S(s-t) x d s .
$$

(iii) For every $x \in W$ and every $t \in\left[t_{0}, t_{1}\right]$ the following equation holds:

$$
\begin{aligned}
P(t) x= & S^{*}\left(t_{1}-t\right) G S\left(t_{1}-t\right) x \\
& +\int_{t}^{t_{1}} S^{*}(s-t)\left[C^{*} C-P(s) B R^{-1} B^{*} P(s)\right] S(s-t) x d s .
\end{aligned}
$$

(iv) For every $x \in Z$ the function $P(t) x, t_{0} \leqq t \leqq t_{1}$ is continuously differentiable with values in $Z^{*}$ and satisfies the differential Riccati equation

$$
\frac{d}{d t} P(t) x+A^{*} P(t) x+P(t) A x-P(t) B R^{-1} B^{*} P(t) x+C^{*} C x=0,
$$

$$
P\left(t_{1}\right) x=G x
$$

In this equation $A$ is regarded as a bounded operator from $Z$ into $V$.

Proof. The equivalence of the statements (i), (ii) and (iii) can be established in a straightforward way using the formulae (2.6) and (2.9) together with Lemma 2.5.

In order to prove that (iii) implies (iv), note that the equation

$$
\langle C S(t) z, C S(t) x\rangle-\langle C z, C x\rangle=\int_{0}^{t}[\langle C S(s) A z, C S(s) x\rangle+\langle C S(s) z, C S(s) A x\rangle] d s
$$

holds for all $x, z \in \mathscr{D}_{W}(A)$ and every $t \geqq 0$. It follows from (H3) and (H2) that both sides of this equation depend continuously on $x, z \in Z=\mathscr{D}_{V}(A) \subset W$ and that $\mathscr{D}_{Z}(A) \subset \mathscr{D}_{W}(A) \subset Z$. Consequently $\mathscr{D}_{W}(A)$ is dense in $Z$ and hence (2.25) holds for all $x, z \in Z$.

From (2.25) we see that the function $\langle z, P(t) x\rangle$-defined by (2.23) -is continuously differentiable on the interval $\left[t_{0}, t_{1}\right]$ for all $x, z \in Z$ and satisfies the equation

$$
\begin{aligned}
\frac{d}{d t}\langle z, P(t) x\rangle= & -\left\langle S\left(t_{1}-t\right) A z, G S\left(t_{1}-t\right) x\right\rangle-\left\langle S\left(t_{1}-t\right) z, G S\left(t_{1}-t\right) A x\right\rangle \\
& -\langle C z, C x\rangle+\left\langle z, P(t) B R^{-1} B^{*} P(t) x\right\rangle \\
& -\int_{t}^{t_{1}}[\langle C S(s-t) A z, C S(s-t) x\rangle \\
& \quad+\langle C S(s-t) z, C S(s-t) A x\rangle] d s \\
& +\int_{t}^{t_{1}}\left\langle S(s-t) A z, P(s) B R^{-1} B^{*} P(s) S(s-t) x\right\rangle d s \\
& +\int_{t}^{t_{1}}\left\langle S(s-t) z, P(s) B R^{-1} B^{*} P(s) S(s-t) A x\right\rangle d s \\
= & -\langle A z, P(t) x\rangle-\langle z, P(t) A x\rangle \\
& -\langle C z, C x\rangle+\left\langle z, P(t) B R^{-1} B^{*} P(t) x\right\rangle .
\end{aligned}
$$


This implies

$$
\begin{aligned}
\langle z, P(t) x\rangle_{Z, Z^{*}}=\left\langle z, G x+\int_{t}^{t_{1}}[\right. & A^{*} P(s) x+P(s) A x \\
& \left.\left.-P(s) B R^{-1} B^{*} P(s) x+C^{*} C x\right] d s\right\rangle_{Z, Z^{*}}
\end{aligned}
$$

and hence (2.24a). Thus we have proved that (iii) implies (iv).

Conversely, let us assume that $P(t)$ satisfies (2.24). Then the following equation holds for every $x \in Z$ and every $t \in\left[t_{0}, t_{1}\right]$

$$
\begin{aligned}
S^{*}\left(t_{1}-t\right) G S\left(t_{1}-t\right) x-P(t) x & =\int_{t}^{t_{1}} \frac{d}{d s} S^{*}(s-t) P(s) S(s-t) x d s \\
& =\int_{t}^{t_{1}} S^{*}(s-t)\left[\dot{P}(s)+A^{*} P(s)+P(s) A\right] S(s-t) x d s \\
& =\int_{t}^{t_{1}} S^{*}(s-t)\left[C^{*} C-P(s) B R^{-1} B^{*} P(s)\right] S(s-t) x d s
\end{aligned}
$$

where the integral has to be understood in the Hilbert space $Z^{*}$ and $\dot{P}(t)$ is the strong derivative of $P(t), t_{0} \leqq t \leqq t_{1}$ regarded as an operator in $\mathscr{L}\left(Z, Z^{*}\right)$.

3. Infinite time control. In this section we consider the control problem of minimizing the performance index

$$
J(u)=\int_{0}^{\infty}\left[\|y(t)\|_{Y}^{2}+\langle u(t), R u(t)\rangle_{U}\right] d t
$$

where $y(t)$ is again the output of (2.1) with $t_{0}=0$, i.e.

$$
y(t)=C S(t) x_{0}+C \int_{0}^{t} S(t-s) B u(s) d s, \quad t \geqq 0 .
$$

For this infinite time problem it is not clear that the cost will be finite for any control input $u(\cdot) \in L^{2}(0, \infty ; U)$. So we add this as another hypothesis.

(H4) For every $x_{0} \in V$ there exists a $u_{x_{0}}(\cdot) \in L^{2}[0, \infty ; U]$ such that $J\left(u_{x_{0}}\right)<\infty$. We will derive the optimal control via the solution of an algebraic Riccati equation which is actually the stationary version of $(2.24)$. For this sake we consider the finite time control problems of minimizing the cost functionals.

$$
J_{T}(u)=\int_{0}^{T}\left[\|y(t)\|_{Y}^{2}+\langle u(t), R u(t)\rangle_{U}\right] d t
$$

subject to the constraint (3.2). The corresponding Riccati operator will be denoted by $P_{T}(t) \in \mathscr{L}\left(V, V^{*}\right)$ and satisfies the equation

$$
P_{T}(t) x=\int_{t}^{T} S^{*}(s-t)\left[C^{*} C-P_{T}(s) B R^{-1} B^{*} P_{T}(s)\right] S(s-t) x d s
$$

for every $x \in W$ and every $t \in[0, T]$.

LEMMA 3.1.

$$
P_{T-\alpha}(t)=P_{T}(t+\alpha), \quad 0 \leqq t \leqq T-\alpha .
$$


Proof. The operator $P_{T}(t+\alpha)$ satisfies the equation

$$
\begin{aligned}
P_{T}(t+\alpha) x & =\int_{t+\alpha}^{T} S^{*}(s-t-\alpha)\left[C^{*} C-P_{T}(s) B R^{-1} B^{*} P_{T}(s)\right] S(s-t-\alpha) x d s \\
& =\int_{t}^{T-\alpha} S^{*}(s-t)\left[C^{*} C-P_{T}(s+\alpha) B R^{-1} B^{*} P_{T}(s+\alpha)\right] S(s-t) x d s
\end{aligned}
$$

for $x \in W$ and $0 \leqq t \leqq T-\alpha$. Thus the statement of the lemma follows from the equivalence of (2.16) and (2.23) (Proposition 2.8) together with the uniqueness result (Theorem 2.7).

We will derive the solution of the algebraic Riccati equation as the limit of the solutions to integral Riccati equations as $T$ goes to infinity. For this we need the following preliminary result which is a special case of Proposition 2.8.

Corollary 3.2. Suppose that the hypotheses $(\mathrm{H} 1),(\mathrm{H} 2)$ and $(\mathrm{H} 3)$ are satisfied and let $P \in \mathscr{L}\left(V, V^{*}\right)$ be a nonnegative, self adjoint operator. Moreover, let $S_{p}(t) \in$ $\mathscr{L}(V) \cap \mathscr{L}(W)$ be the strongly continuous semigroup which is generated by $A-$ $B R^{-1} B^{*} P: \mathscr{D}_{V}(A) \rightarrow V$, i.e. $S_{p}(t)$ satisfies the equation

$$
S_{p}(t) x=S(t) x-\int_{0}^{t} S(t-s) B R^{-1} B^{*} P S_{p}(s) x d s
$$

for $x \in V$ and $t \geqq 0$. Then the following statements are equivalent.

(i) For every $x \in W$ and every $t \geqq 0$

$$
P x=S_{p}^{*}(t) P S_{p}(t) x+\int_{0}^{t} S_{p}^{*}(s)\left[C^{*} C+P B R^{-1} B^{*} P\right] S_{p}(s) x d s .
$$

(ii) For every $x \in W$ and every $t \geqq 0$

$$
P x=S_{p}^{*}(t) P S(t) x+\int_{0}^{t} S_{p}^{*}(s) C^{*} C S(s) x d s .
$$

(iii) For every $x \in W$ and every $t \geqq 0$

$$
P x=S^{*}(t) P S(t) x+\int_{0}^{t} S^{*}(s)\left[C^{*} C-P B R^{-1} B^{*} P\right] S(s) x d s .
$$

(iv) For every $x \in Z$ the following equation holds in $Z^{*}$

$$
A^{*} P x+P A x-P B R^{-1} B^{*} P x+C^{*} C x=0 .
$$

Now we are in the position to prove the main result of this section.

THEOREM 3.3. Let (H1), (H2) and (H3) be satisfied. Then the following statements hold.

(i) The hypothesis (H4) is satisfied if and only if there exists a nonnegative self adjoint solution $P \in \mathscr{L}\left(V, V^{*}\right)$ of (3.9).

(ii) If (H4) is satisfied, then there exists a unique optimal control $u_{p}(\cdot) \in L^{2}(0, \infty ; U)$ which is given by the feedback law.

$$
u_{p}(t)=-R^{-1} B^{*} P x(t), \quad t \geqq 0,
$$

where $P \in \mathscr{L}\left(V, V^{*}\right)$ is the (unique) minimal solution of (3.9). Moreover, the optimal cost is given by

$$
J\left(u_{p}\right)=\left\langle x_{0}, P x_{0}\right\rangle .
$$

(iii) If (H4) is satisfied, then the minimal solution $P \in \mathscr{L}\left(V, V^{*}\right)$ of (3.9) is strong limit of $P_{T}(0) \in \mathscr{L}\left(V, V^{*}\right)$ as $T$ goes to infinity where $P_{T}(t)$ is defined by (3.4). 
Proof. First recall that the optimal control of the finite time problem on the interval $[0, T]$ is given by $u_{T}(t)=-R^{-1} B^{*} P_{T}(t) x(t), 0 \leqq t \leqq T$, and the optimal cost by $J_{T}\left(u_{T}\right)=$ $\left\langle x_{0}, P_{T}(0) x_{0}\right\rangle$ (Theorem 2.7). So (H4) implies that

$$
\left\langle x_{0}, P_{T}(0) x_{0}\right\rangle=J_{T}\left(u_{T}\right) \leqq J_{T}\left(u_{x_{0}}\right) \leqq J\left(u_{x_{0}}\right)<\infty
$$

and thus there exists a limit of the increasing function $\left\langle x_{0}, P_{T}(0) x_{0}\right\rangle, T \geqq 0$, for every $x_{0} \in V$. Hence there exists a nonnegative, self adjoint operator $P \in \mathscr{L}\left(V, V^{*}\right)$ which is the strong limit of $P_{T}(0)$ (Kato [15, p. 454, Thm. 3.3], compare the proof of Theorem 2.7).

By Lemma 3.1,

$$
P x=s-\lim _{T \rightarrow \infty} P_{T}(t) x \in V^{*}
$$

exists uniformly in $t$ on every compact time interval. Making use of formula (3.4), we obtain for $x \in W$ and $t \geqq 0$

$$
\begin{aligned}
P x= & \lim _{T \rightarrow \infty} P_{T}(0) x \\
= & \lim _{T \rightarrow \infty} \int_{0}^{T} S^{*}(s)\left[C^{*} C-P_{T}(s) B R^{-1} B^{*} P_{T}(s)\right] S(s) x d s \\
= & \lim _{T \rightarrow \infty} \int_{t}^{T} S^{*}(t) S^{*}(s-t)\left[C^{*} C-P_{T}(s) B R^{-1} B^{*} P_{T}(s)\right] S(s-t) S(t) x d s \\
& +\lim _{T \rightarrow \infty} \int_{0}^{t} S^{*}(s)\left[C^{*} C-P_{T}(s) B R^{-1} B^{*} P_{T}(s)\right] S(s) x d s \\
= & \lim _{T \rightarrow \infty} S^{*}(t) P_{T}(t) S(t) x+\int_{0}^{t} S^{*}(s)\left[C^{*} C-P B R^{-1} B^{*} P\right] S(s) x d s \\
= & S^{*}(t) P S(t) x+\int_{0}^{t} S^{*}(s)\left[C^{*} C-P B R^{-1} B^{*} P\right] S(s) x d s
\end{aligned}
$$

and hence $P \in \mathscr{L}\left(V, V^{*}\right)$ is a solution of (3.6), (3.7), (3.8) and (3.9).

Conversely, let $Q \in \mathscr{L}\left(V, V^{*}\right)$ be any nonnegative solution of (3.9) and let $u_{Q}(t)=$ $-R^{-1} B^{*} Q x(t)$ be the corresponding feedback control law with the associated closed loop semigroup $S_{Q}(t) \in \mathscr{L}(V) \cap \mathscr{L}(W)$. Then the following inequality holds for every $x_{0} \in V$

$$
\begin{aligned}
\left\langle x_{0}, Q x_{0}\right\rangle= & \lim _{t \rightarrow \infty}\left\{\left\langle S_{Q}(t) x_{0}, Q S_{Q}(t) x_{0}\right\rangle\right. \\
& \left.\quad+\int_{0}^{t}\left\langle S_{Q}(s) x_{0},\left[C^{*} C+Q B R^{-1} B^{*} Q\right] S_{Q}(s) x_{0}\right\rangle d s\right\} \\
\geqq & \int_{0}^{\infty}\left\langle S_{Q}(t) x_{0},\left[C^{*} C+Q B R^{-1} B^{*} Q\right] S_{Q}(s) x_{0}\right\rangle d s \\
= & J\left(u_{Q}\right)
\end{aligned}
$$

and hence (H4) is satisfied. Moreover, the operator $P \in \mathscr{L}\left(V, V^{*}\right)$ defined by (3.12) satisfies the inequality

$$
\left\langle x_{0}, P x_{0}\right\rangle=\lim _{T \rightarrow \infty}\left\langle x_{0}, P_{T}(0) x_{0}\right\rangle \leqq \lim _{T \rightarrow \infty} J_{T}(u)=J(u)
$$


for every admissible control $u(\cdot) \in L^{2}(0, \infty ; U)$. This shows that $P$ is the minimal positive semidefinite solution of (3.6). Finally, taking $Q=P$, we conclude that the unique optimal control is given by (3.10) with cost (3.11).

Although the above theorem yields a solution to the infinite time problem, in a sense it is unsatisfactory. This is because we are not sure of a unique solution to the algebraic Riccati equation and also we cannot be sure that the semigroup $S_{p}(t)$ is exponentially stable. In order to resolve those difficulties, we need another hypothesis.

(H5) If $x_{0} \in V$ and $u(\cdot) \in L^{2}(0, \infty ; U)$ are such that $J(u)<\infty$, then $x(\cdot) \in$ $L^{2}(0, \infty ; V)$ where $x(t), t \geqq 0$, is given by (2.3) with $t_{0}=0$.

Theorem 3.4. Let (H1), (H2), (H3) and (H5) be satisfied. Then the algebraic Riccati equation (3.9) has at most one nonnegative, self adjoint solution $P \in \mathscr{L}\left(V, V^{*}\right)$. Moreover, if $P$ is such a solution, then the closed loop semigroup $S_{p}(t) \in \mathscr{L}(V)$ is exponentially stable.

Proof. If $P \in \mathscr{L}\left(V, V^{*}\right)$ is a positive semidefinite solution of (3.9), then the inequality (3.13) with $Q=P$ shows that the closed loop control $u_{p}(t)=-R^{-1} B^{*} P x(t)$ has a finite cost for every initial state $x_{0} \in V$. By hypothesis (H5) this means that

$$
\int_{0}^{\infty}\left\|S_{p}(t) x_{0}\right\|_{V}^{2} d t<\infty
$$

for every $x_{0} \in V$. Hence it follows from a result of Datko [5] that the semigroup $S_{p}(t) \in \mathscr{L}(V)$ is exponentially stable (see Curtain and Pritchard [4]). The stability of $S_{p}(t)$ shows that we have equality in (3.13) and hence

$$
J\left(u_{p}\right)=\left\langle x_{0}, P x_{0}\right\rangle .
$$

Now let $Q \in \mathscr{L}\left(V, V^{*}\right)$ be another nonnegative solution of (3.9) and let us apply Lemma 2.6 to the performance index

$$
J_{T, Q}(u)=\langle x(T), Q x(T)\rangle+\int_{0}^{T}\left[\|y(t)\|_{Y}^{2}+\langle u(t), R u(t)\rangle\right] d t
$$

as well as the feedback $F(t) \equiv-R^{-1} B^{*} Q$ and the control input $u_{p}(t)$. Then $P_{F}(t) \equiv Q$ and hence the inequality

$$
\begin{aligned}
\left\langle x_{0}, P x_{0}\right\rangle & =J\left(u_{p}\right)=\lim _{T \rightarrow \infty} J_{T, Q}\left(u_{p}\right) \\
& =\lim _{T \rightarrow \infty}\left[\left\langle x_{0}, Q x_{0}\right\rangle+\int_{0}^{T}\left\langle R^{-1} B^{*} Q x(t)+u_{p}(t), R\left[R^{-1} B^{*} Q x(t)+u_{p}(t)\right]\right\rangle d t\right] \\
& \geqq\left\langle x_{0}, Q x_{0}\right\rangle
\end{aligned}
$$

holds for every $x_{0} \in V$. Interchanging the roles of $P$ and $Q$, we conclude that $P=Q$.

Finally, let us briefly discuss the hypotheses (H4) and (H5) which are chosen in a general sense but are difficult to check in concrete examples. In most cases it might be desirable to replace them by stronger assumptions which are easier to check.

Remarks 3.5. Let (H1) and (H2) be satisfied.

(i) Suppose that system (2.1) is stabilizable in the sense that there exists a feedback operator $F \in \mathscr{L}(V, U)$ such that the closed loop semigroup $S_{F}(t) \in \mathscr{L}(V)$ defined by

$$
S_{F}(t) x=S(t) x+\int_{0}^{t} S(t-s) B F S_{F}(s) x d s
$$

for $t \geqq 0$ and $x \in V$ is exponentially stable. Then hypothesis (H4) is satisfied. 
In fact, there is an instant $T>0$ and a constant $c_{T}>0$ such that the inequalities

$$
\left\|S_{F}(T)\right\|_{\mathscr{L}(V)}<1, \quad\left\|C S_{F}(\cdot) x\right\|_{L^{2}(0, T ; Y)} \leqq c_{T}\|x\|_{V}
$$

hold for every $x \in W$. This implies that

$$
\left\|C S_{F}(\cdot) x\right\|_{L^{2}(0, \infty ; Y)} \leqq c_{T} \sum_{k=0}^{\infty}\left\|S_{F}(T)\right\|_{\mathscr{L}(V)}^{k}\|x\|_{V}
$$

for $x \in W$ and hence (H4) is satisfied.

(ii) Suppose that system (2.1) is detectable in the sense that there exists an operator $K \in \mathscr{L}(Y, V)$ such that the output injection semigroup $S_{K}(t) \in \mathscr{L}(V)$ defined by

$$
S_{K}(t) x=S(t) x+\int_{0}^{t} S_{K}(t-s) K C S(s) x d s
$$

for $t \geqq 0$ and $x \in W$ (see Salamon [25, Thm. I.3.9]) is exponentially stable. Then hypothesis ( $\mathrm{H} 5)$ is satisfied.

In fact, if $x(t) \in V$ and $y(t) \in Y$ are defined by (2.3) for $x_{0} \in V$ and $u(\cdot) \in$ $L_{\text {loc }}^{2}(0, \infty ; U)$, then it is easy to see

$$
x(t)=S_{K}(t) x_{0}+\int_{0}^{t} S_{K}(t-s)[B u(s)-K y(s)] d s, \quad t \geqq 0 .
$$

Hence $J(u)<\infty$ implies that $x(\cdot) \in L^{2}(0, \infty ; V)$.

(iii) If (H4) and (H5) are satisfied, then system (2.1) is stabilizable in the sense of (i). (Theorems 3.3 and 3.4.)

(iv) For finite dimensional systems (H5) is equivalent to detectability in the sense of (ii). It seems to be an open problem whether this equivalence extends to the infinite dimensional situation.

\section{Examples.}

4.1. Neutral systems with output delays. We consider the linear neutral functional differential equation (NFDE)

$$
\frac{d}{d t}\left(x(t)-M x_{t}\right)=L x_{t}+B_{0} u(t), \quad y(t)=C x_{t},
$$

where $x(t) \in \mathbb{R}^{n}, u(t) \in \mathbb{R}^{m}, y(t) \in \mathbb{R}^{p}$ and $x_{t}$ is defined by $x_{t}(\tau)=x(t+\tau),-h \leqq \tau \leqq 0$, $h>0 . B_{0}$ is an $n \times m$ matrix and $L, M, C$ are bounded linear functionals from $\mathscr{C}=\mathscr{C}\left[-h, 0 ; \mathbb{R}^{n}\right]$ into $\mathbb{R}^{n}$ and $\mathbb{R}^{p}$ respectively. These can be represented by matrixfunctions $\eta(\tau), \mu(\tau), \gamma(\tau)$ of bounded variation in the following way

$$
\begin{array}{rlrl}
L \phi & =\int_{-h}^{0} d \eta(\tau) \phi(\tau), & & M \phi=\int_{-h}^{0} d \mu(\tau) \phi(\tau), \\
C \phi & =\int_{-h}^{0} d \gamma(\tau) \phi(\tau), & \phi \in \mathscr{C} .
\end{array}
$$

In order to guarantee the existence and uniqueness of solutions of (4.1), we will always assume

$$
\mu(0)=\lim _{\tau \uparrow 0} \mu(\tau) .
$$

Moreover, we will assume at some places that $M: \mathscr{C} \rightarrow \mathbb{R}^{n}$ is of the special form

$$
M \phi=\sum_{j=1}^{\infty} A_{-j} \phi\left(-h_{j}\right)+\int_{-h}^{0} A_{-\infty}(\tau) \phi(\tau) d \tau, \quad \phi \in \mathscr{C},
$$

where $0<h_{j} \leqq h, A_{-j} \in \mathbb{R}^{n \times n}$ for $j \in \mathbb{N}, A_{-\infty}(\cdot) \in L^{1}\left[-h, 0 ; \mathbb{R}^{n \times n}\right]$ and $\sum_{j=1}^{\infty}\left\|A_{-j}\right\|<\infty$. 
A function $x(\cdot) \in L_{\text {loc }}^{2}\left(-h, \infty ; \mathbb{R}^{n}\right)$ is said to be a solution of (4.1) if the function $w(t)=x(t)-M x_{t}$ is absolutely continuous with an $L^{2}$-derivative on every compact interval $[0, T], T>0$, and if $\dot{w}(t)=L x_{t}+B_{0} u(t)$ for almost every $t \geqq 0$. It is well known (Burns, Herdman and Stech [3], Salamon [25]) that (4.1) admits a unique solution $x(t), t \geqq-h$, for every input $u(\cdot) \in L_{\text {loc }}^{2}\left(0, \infty ; \mathbb{R}^{m}\right)$ and every initial condition

$$
\lim _{t \downarrow 0} x(t)-M x_{t}=\phi^{0}, \quad x(\tau)=\phi^{1}(\tau), \quad-h \leqq \tau<0,
$$

where $\phi=\left(\phi^{0}, \phi^{1}\right) \in M^{2}=\mathbb{R}^{n} \times L^{2}\left(-h, 0 ; \mathbb{R}^{n}\right)$. Moreover it has been shown in [3], [25] that the evolution of the state

$$
\hat{x}(t)=\left(x(t)-M x_{t}, x_{t}\right) \in M^{2}
$$

of system (4.1), (4.4) can be described by the formula

$$
\hat{x}(t)=S(t) \phi+\int_{0}^{t} S(t-s) B u(s) d s
$$

where $B \in \mathscr{L}\left(\mathbb{R}^{m}, M^{2}\right)$ maps $u \in \mathbb{R}^{m}$ into the pair $B u=\left(B_{0} u, 0\right)$ and $S(t) \in \mathscr{L}\left(M^{2}\right)$ is the strongly continuous semigroup generated by $A$, where

$$
D(A)=\left\{\phi \in M^{2}: \phi^{1} \in W^{1,2}, \phi^{0}=\phi^{1}(0)-M \phi^{1}\right\}, \quad A \phi=\left(L \phi^{1}, \dot{\phi}^{1}\right) .
$$

Here $W^{1,2}$ denotes the Sobolev space $W^{1,2}\left(-h, 0 ; \mathbb{R}^{n}\right)$.

Obviously, the dense subspace

$$
W=\left\{(\phi(0)-M \phi, \phi): \phi \in W^{1,2}\right\}=\mathscr{D}(A)
$$

of $M^{2}$-endowed with the $W^{1,2}$ norm-is invariant under $S(t)$ and $S(t)$ can be restricted to a strongly continuous semigroup on $W$.

The output of the system (4.1) may be described through the operator

$$
C: W \rightarrow \mathbb{R}^{p}, \quad C \phi=\int_{-h}^{0} d \gamma(\tau) \phi^{1}(\tau), \quad \phi \in W .
$$

Remarks 4.1. (i) The infinitesimal generator $A$ of $S(t)$ can be interpreted as a bounded operator from $W$ into $M^{2}$. By duality, $M^{2}$ can be regarded as a dense subspace of $W^{*}$ and $A^{*}$ extends to a bounded operator from $M^{2}$ into $W^{*}$.

(ii) It has been proved in Burns, Herdman and Stech [3] and Salamon [25] that system (4.1) satisfies the hypotheses (H1) and (H2) with $H=V=M^{2}$ and the subspace $W \subset M^{2}$ as defined above. Hypothesis (H1) says that the state $\hat{x}(T) \in M^{2}$ of (4.1) defined by $(4.5)$ is in $W$ for every input $u(\cdot) \in L^{2}\left(0, T ; \mathbb{R}^{m}\right)$ and zero initial condition and that $\hat{x}(T) \in W$ depends continuously on $u(\cdot) \in L^{2}\left[0, T ; \mathbb{R}^{m}\right]$. Hypothesis (H2) says that the output $y(\cdot)$ of the free system (4.1) (i.e. $u(t) \equiv 0)$ is in $L^{2}\left(0, T ; \mathbb{R}^{p}\right)$ and depends in this space continuously on the initial state $\phi \in M^{2}$.

(iii) If $M: \mathscr{C} \rightarrow \mathbb{R}^{n}$ is given by (4.3), then it is known that the semigroup $S(t) \in$ $\mathscr{L}\left(M^{2}\right)$ is exponentially stable if and only if

$$
\omega_{0}=\sup \{\operatorname{Re} \lambda: \operatorname{det} \Delta(\lambda)=0\}<0
$$

where $\Delta(\lambda)=\lambda\left[I-M\left(e^{\lambda \cdot}\right)\right]-L\left(e^{\lambda \cdot}\right), \lambda \in \mathbb{C}$, is the characteristic matrix of the NFDE (4.1). A necessary condition for the exponential stability of $S(t)$ is the stability of the difference operator which means that

$$
\sup \left\{\operatorname{Re} \lambda: \operatorname{det}\left[I-\sum_{j=1}^{\infty} A_{-j} e^{-\lambda h_{j}}\right]=0\right\}<0 .
$$


These facts have been established by Henry [11] for $S(t) \in \mathscr{L}(W)$. They extend to $S(t) \in \mathscr{L}\left(M^{2}\right)$ because of the similarity of these two semigroups through the transformation $\mu I-A: W \rightarrow M^{2}$ with $\mu \notin \sigma(A)$.

(iv) If $M: \mathscr{C} \rightarrow \mathbb{R}^{n}$ is given by (4.3) and if (4.7) holds, then system (4.1) is stabilizable in the sense that there exists a feedback operator $F \in \mathscr{L}\left(-M^{2}, \mathbb{R}^{m}\right)$ such that the closed loop semigroup $S_{F}(t) \in \mathscr{L}\left(M^{2}\right)$ generated by $A+B F$ is exponentially stable if and only if

$$
\operatorname{rank}\left[\Delta(\lambda), B_{0}\right]=n \quad \forall \lambda \in \mathbb{C}, \quad \operatorname{Re} \lambda \geqq 0
$$

(Pandolfi [21], Salamon [25]).

(v) If $M: \mathscr{C} \rightarrow \mathbb{R}^{n}$ is given by (4.3) and if (4.7) holds, then system (4.1) is detectable in the sense that there exists an output injection operator $K \in \mathscr{L}\left(\mathbb{R}^{p}, M^{2}\right)$ such that the closed loop semigroup $S_{K}(t) \in \mathscr{L}\left(M^{2}\right)$ generated by $A+K C$ is exponentially stable if and only if

$$
\operatorname{rank}\left[\begin{array}{c}
\Delta(\lambda) \\
C\left(e^{\lambda \cdot}\right)
\end{array}\right]=n \quad \forall \lambda \in \mathbb{C}, \quad \operatorname{Re} \lambda \geqq 0
$$

(Salamon [25]).

Associated with the system (4.1) we consider the performance index

$$
J(u)=\int_{0}^{\infty}\left[\|y(t)\|_{\mathbb{R}^{p}}^{2}+\|u(t)\|_{\mathbb{R}^{m}}^{2}\right] d t .
$$

Then we have the following theorem (compare Ito and Tarn [14] and Datko [7]).

Theorem 4.2. Assume $M: \mathscr{C} \rightarrow \mathbb{R}^{n}$ is given by (4.3) and (4.7) is satisfied; then the following statements hold.

(i) If (4.8) is satisfied, there exists, for every initial state $\phi \in M^{2}$, a unique optimal control which minimizes the cost functional (4.10). This optimal control is given by the feedback law

$$
u_{\pi}(t)=-B^{*} \pi \hat{x}(t)
$$

where $\pi \in \mathscr{L}\left(M^{2}\right)$ is the minimal selfadjoint, nonnegative operator which satisfies the algebraic Riccati equation

$$
A^{*} \pi+\pi A+C^{*} C-\pi B B^{*} \pi=0
$$

(this equation must be understood in the space $\mathscr{L}\left(W, W^{*}\right)$ ). Moreover the optimal cost is given by

$$
J\left(u_{\pi}\right)=\langle\phi, \pi \phi\rangle_{M^{2}} .
$$

(ii) If (4.9) is satisfied, then there exists at most one nonnegative self adjoint solution $\pi \in \mathscr{L}\left(M^{2}\right)$ of (4.12). Moreover if $\pi$ is such a solution, the closed loop semigroup $S_{\pi}(t) \in \mathscr{L}\left(M^{2}\right)$ generated by $A-B B^{*} \pi$ is exponentially stable.

4.2. Parabolic systems. Consider the system

$$
\dot{x}=A x+B u, \quad y=C x
$$

where $A$ is a self adjoint operator on a real Hilbert space $H$. We assume that $A$ has a compact resolvent operator and that the spectrum of $A$ consists of a strictly decreasing sequence $\lambda_{n}, n \in \mathbb{N}$, of real eigenvalues with associated eigenvector $\phi_{n} \in H,\left\|\phi_{n}\right\|=1$. Then $A$ generates the strongly continuous semigroup $S(t)$ on $H$ given by

$$
S(t) x=\sum_{n=1}^{\infty} e^{\lambda_{n} t}\left\langle x, \phi_{n}\right\rangle \phi_{n} .
$$


We assume that $W$ and $V$ are given by

$$
\begin{aligned}
& W=\left\{x \in H \mid \sum_{n=1}^{\infty} \gamma_{n}\left\langle x, \phi_{n}\right\rangle^{2}<\infty\right\}, \\
& V^{*}=\left\{x \in H \mid \sum_{n=1}^{\infty} \beta_{n}^{-1}\left\langle x, \phi_{n}\right\rangle^{2}<\infty\right\}
\end{aligned}
$$

with the obvious inner products, where $\beta_{n}$ and $\gamma_{n}$ are positive sequences satisfying $0<\beta_{n} \leqq 1 \leqq \gamma_{n}<\infty$ for $n \in \mathbb{N}$. Then the space $V$ can be represented as a space of sequences in the following way

$$
V=\left\{x \in \mathbb{R}^{\mathbb{N}} \mid \sum_{n=1}^{\infty} \beta_{n} x_{n}^{2}<\infty\right\}
$$

and the injection $H \subset V$ is given by identifying $x \in H$ with the sequence $\left\{\left\langle x, \phi_{n}\right\rangle\right\}_{n \subset \mathbb{N}} \in V$. Finally, we assume that the sequences $b_{n} \in U, c_{n} \in Y$ satisfy

$$
\sum_{n=1}^{\infty} \beta_{n}\left\|b_{n}\right\|_{U}^{2}<\infty, \quad \sum_{n=1}^{\infty} \gamma_{n}^{-1}\left\|c_{n}\right\|_{Y}^{2}<\infty
$$

and that the operators $B \in \mathscr{L}(U, V)$ and $C \in \mathscr{L}(W, Y)$ are given by

$$
C x=\sum_{n=1}^{\infty} c_{n}\left\langle x, \phi_{n}\right\rangle, \quad B u=\left\{\left\langle b_{n}, u\right\rangle_{U}\right\}_{n \in \mathbb{N}} .
$$

LEMMA 4.3. (i) Let $n_{0}=\max \left\{n \in \mathbb{N} \mid \lambda_{n} \geqq 0\right\}$ and suppose that

$$
\sum_{n=n_{0}+1}^{\infty} \frac{\gamma_{n}\left\|b_{n}\right\|^{2}}{\left|\lambda_{n}\right|}<\infty
$$

then hypothesis $(\mathrm{H} 1)$ is satisfied.

(ii) If

$$
\sum_{n=n_{0}+1}^{\infty} \frac{\left\|c_{n}\right\|^{2}}{\beta_{n}\left|\lambda_{n}\right|}<\infty
$$

then hypothesis $(\mathrm{H} 2)$ is satisfied.

Proof. Statement (ii) is the dual of (i) and statement (i) follows from the inequality

$$
\begin{aligned}
\left\|\int_{0}^{T} S(t-s) B u(s) d s\right\|_{W} & =\sum_{n=1}^{\infty} \gamma_{n}\left(\int_{0}^{T} e^{\lambda_{n}(T-s)} b_{n} u(s) d s\right)^{2} \\
& \leqq \sum_{n=1}^{\infty} \gamma_{n} \int_{0}^{T} e^{2 \lambda_{n} s} d s\left\|b_{n}\right\|^{2}\|u(\cdot)\|_{L^{2}[0, T ; U]}^{2} \\
& \leqq\left[\sum_{n=1}^{n_{0}} \gamma_{n} \int_{0}^{T} e^{2 \lambda_{n} s} d s\left\|b_{n}\right\|^{2}+\sum_{n=n_{0}+1}^{\infty} \frac{\gamma_{n}\left\|b_{n}\right\|^{2}}{2\left|\lambda_{n}\right|}\right]\|u(\cdot)\|_{L^{2}[0, T ; U]}^{2}
\end{aligned}
$$

In concrete examples the sequences $b_{n}, c_{n}$ are given and the spaces $W$ and $V$ have to be chosen in such a way that $(\mathrm{H} 1),(\mathrm{H} 2)$ and $(\mathrm{H} 3)$ are satisfied. The next lemma shows under which conditions this is possible.

LeMMA 4.4. Let the sequences $b_{n} \in U, c_{n} \in Y, \lambda_{n} \in \mathbb{R}$ be given such that $\lambda_{n}$ is strictly decreasing and tends to $-\infty$. Then there exist positive sequences $\beta_{n}, \gamma_{n}$ satisfying (4.15)(4.17) if and only if

$$
\sum_{n=n_{0}+1}^{\infty} \frac{\left\|b_{n}\right\| \cdot\left\|c_{n}\right\|}{\left|\lambda_{n}\right|^{1 / 2}}<\infty
$$


Furthermore, if (4.18) holds, then the sequences $\beta_{n}, \gamma_{n}$ can be chosen such that $\beta_{n} \leqq \gamma_{n} \leqq$ $\beta_{n}\left|\lambda_{n}\right|$ for almost every $n \in \mathbb{N}$.

Proof. The necessity of (4.18) is obvious. Conversely if (4.18) holds, then it is easy to see that the sequences

$$
\begin{gathered}
\beta_{n}=\left\{\begin{array}{lll}
\left\|c_{n}\right\| /\left\|b_{n}\right\|\left|\lambda_{n}\right|^{1 / 2}, & b_{n} \neq 0, \quad c_{n} \neq 0, \quad \lambda_{n} \neq 0, \\
n^{2}\left\|c_{n}\right\|^{2} /\left|\lambda_{n}\right|, & b_{n}=0, \quad c_{n} \neq 0, \quad \lambda_{n} \neq 0, \\
1 / n^{2}\left\|b_{n}\right\|^{2}, & b_{n} \neq 0, \quad c_{n}=0, \quad \lambda_{n} \neq 0, \\
1 & \text { otherwise, }
\end{array}\right. \\
\gamma_{n}=\left\{\begin{array}{lll}
\left|\lambda_{n}\right|^{1 / 2}\left\|c_{n}\right\| /\left\|b_{n}\right\|, & b_{n} \neq 0, \quad c_{n} \neq 0, \quad \lambda_{n} \neq 0, \\
n^{2}\left\|c_{n}\right\|^{2}, & b_{n}=0, \quad c_{n} \neq 0, \quad \lambda_{n} \neq 0, \\
\left|\lambda_{n}\right| / n^{2}\left\|b_{n}\right\|^{2}, & b_{n} \neq 0, \quad c_{n}=0, \quad \lambda_{n} \neq 0, \\
\max \left\{1,\left|\lambda_{n}\right|\right\} & \text { otherwise, }
\end{array}\right.
\end{gathered}
$$

satisfy the requirements of the lemma.

Remarks 4.5. (i) The condition $\gamma_{n}=\beta_{n}\left|\lambda_{n}\right|$ for almost every $n \in \mathbb{N}$ (with at most a finite number of exceptions) means that

$$
\mathscr{D}_{V}(A) \subset \mathscr{D}_{V}\left((-A)^{1 / 2}\right)=\left\{x \in V\left|\sum_{n=1}^{\infty} \beta_{n}\right| \lambda_{n} \mid x_{n}^{2}<\infty\right\}=W \subset V
$$

so that $(\mathrm{H} 3)$ is satisfied.

(ii) We can assume without loss of generality that $W \subset H \subset V$, i.e. the sequences $\beta_{n}$ and $\gamma_{n}^{-1}$ are bounded. This can always be achieved by redefining $b_{n}, c_{n}, \beta_{n}$ and $\gamma_{n}$.

(iii) It is well known that system (4.14) is stabilizable in the space $V$ if and only if $b_{n} \neq 0$ for $n=1, \cdots, n_{0}$ (Curtain and Pritchard [4]).

The system is detectable through the unbounded output operator $C: W \rightarrow Y$ if and only if $c_{n} \neq 0$ for $n=1, \cdots, n_{0}$. This follows from an obvious generalization of the standard result for bounded output operators using a perturbation result in Salamon [25].

We are now in the position to apply the Theorems 3.3 and 3.4 to the Cauchy problem (4.14) with the performance index (4.10). Hence there exists a unique nonnegative operator $P \in \mathscr{L}\left(V, V^{*}\right)$ satisfying the algebraic Riccati equation.

$$
A P+P A-P B B^{*} P+C^{*} C=0
$$

if $b_{n} \neq 0$ and $c_{n} \neq 0$ for $n=1, \cdots, n_{0}$. Furthermore the optimal control is given by the feedback law

$$
u(t)=-B^{*} P x(t)
$$

Example 4.6. As a specific example consider

$$
\begin{aligned}
& z_{t}=z_{\xi \xi}, \quad 0<\xi<1, \\
& z_{\xi}(t, 0)=u(t), \quad z_{\xi}(t, 1)=0, \quad t>0, \\
& y(t)=\int_{0}^{1} c(\xi) z(t, \xi) d \xi, \quad t>0 .
\end{aligned}
$$

It can be shown (see Curtain and Pritchard [4]) that this system is equivalent to a Cauchy problem of the form (4.14) with $H=L^{2}[0,1], \lambda_{0}=0, \phi_{0}(\xi) \equiv 1, \lambda_{n}=-n^{2} \pi^{2}$, $\phi_{n}(\xi)=\sqrt{2} \cos n \pi \xi$, and $B u=-\delta u$ ( $\delta$ being the Dirac delta impulse at $\left.\xi=0\right)$. Hence 
we get $b_{0}=-1, b_{n}=-\sqrt{2}$ for $n \in \mathbb{N}$ and $c_{n}=\left\langle c, \phi_{n}\right\rangle$ for $n=0,1,2, \cdots$. So condition (4.18) is satisfied if and only if

$$
\sum_{n=1}^{\infty} \frac{\left|c_{n}\right|}{n}<\infty
$$

This allows for arbitrary bounded linear output operators $C: L^{2}[0,1] \rightarrow \mathbb{R}$ and even for a class of unbounded output operators. If $C$ is bounded, then $c_{n}$ is square summable and we may choose $\gamma_{n}=1, \beta_{n}=n^{-2}$, which means that

$$
W=L^{2}[0,1], \quad V^{*}=H^{1}[0,1] .
$$

Remark 4.7. Existence and uniqueness results for the differential Riccati equation associated with parabolic systems have been established by Pritchard and Pollock [22], Flandoli [9] and Sorine [26], [27] under weaker hypothesis. The assumptions in these papers are, roughly speaking, that $A$ is a self adjoint nonpositive operator on $H$ and that

$$
W=V^{*}=\mathscr{D}\left((-A)^{1 / 2}\right) .
$$

In [9] and [22] it is assumed that the function $\|C S(t) B\|_{\mathscr{L}(U, V)}$ is integrable on $[0, T]$, whereas our results are only applicable if this function is square integrable. However, in [9] and [22] the Riccati operator $P(t)$ will only be in $\mathscr{L}(V, H) \cap \mathscr{L}\left(H, V^{*}\right)$ and correspondingly the optimal feedback operator $F(t)=-B^{*} P(t)$ will only be in $\mathscr{L}(H, U)$ as opposed to $\mathscr{L}(V, U)$.

4.3. Hyperbolic systems. Consider the system

$$
\ddot{z}=A z+B u, \quad y=C z,
$$

where $A$ is a self adjoint operator on a real Hilbert space $H$. We assume that $A$ has a compact resolvent operator and that its (simple) negative eigenvalues $\lambda_{n}=-\omega_{n}^{2}$ satisfy

$$
\omega_{1} \geqq \delta, \quad \omega_{n+1}-\omega_{n} \geqq \delta, \quad n \in \mathbb{N},
$$

for some $\delta>0$. The corresponding eigenvectors are denoted by $\phi_{n} \in H,\left\|\phi_{n}\right\|=1$. Furthermore, we assume that the spaces $W_{0}, V_{1}$ are given by

$$
\begin{aligned}
& W_{0}=\left\{x \in H\left|\sum_{n=1}^{\infty} \gamma_{n}\right| \lambda_{n} \mid\left\langle x, \phi_{n}\right\rangle^{2}<\infty\right\}, \\
& V_{1}^{*}=\left\{x \in H \mid \sum_{n=1}^{\infty} \beta_{n}^{-1}\left\langle x, \phi_{n}\right\rangle^{2}<\infty\right\},
\end{aligned}
$$

where $\beta_{n}$ and $\gamma_{n}$ are positive sequences satisfying $0 \leqq \beta_{n} \leqq 1 \leqq \gamma_{n} \leqq \beta_{n}\left|\lambda_{n}\right|$ for $n \in \mathbb{N}$. Finally, we assume that the sequences $b_{n} \in U, c_{n} \in Y$ satisfy

$$
\sum_{n=1}^{\infty} \beta_{n}\left\|b_{n}\right\|^{2}<\infty, \quad \sum_{n=1}^{\infty} \frac{\left\|c_{n}\right\|^{2}}{\gamma_{n}\left|\lambda_{n}\right|}<\infty,
$$

and that the operators $B \in \mathscr{L}\left(U, V_{1}\right), C \in \mathscr{L}\left(W_{0}, Y\right)$ are given by

$$
B^{*} x=\sum_{n=1}^{\infty}\left\langle x, \phi_{n}\right\rangle b_{n}, \quad C x=\sum_{n=1}^{\infty}\left\langle x, \phi_{n}\right\rangle c_{n}
$$

Defining $V \subset H$ by

$$
V=\mathscr{D}\left((-A)^{1 / 2}\right)=\left\{x \in H\left|\sum_{n=1}^{\infty}\right| \lambda_{n} \mid\left\langle x, \phi_{n}\right\rangle^{2}<\infty\right\}
$$


and identifying $H$ with its dual, we obtain that $V \subset H \subset V^{*}$ and $A$ extends to a bounded operator from $V$ to $V^{*}$. In order to transform (2.22) into a first order system, we introduce the product space

$$
\mathscr{H}=V \times H
$$

with inner product

$$
\langle x, \hat{x}\rangle=\sum_{n=1}^{\infty}\left[\left|\lambda_{n}\right|\left\langle x_{0}, \phi_{n}\right\rangle\left\langle\hat{x}, \phi_{n}\right\rangle+\left\langle x_{1}, \phi_{n}\right\rangle\left\langle\hat{x}_{1}, \phi_{n}\right\rangle\right] .
$$

Then the operator $\mathscr{A}: \mathscr{D}(\mathscr{A}) \rightarrow \mathscr{H}$ defined by

$$
\mathscr{A}=\left[\begin{array}{ll}
0 & I \\
A & 0
\end{array}\right], \quad \mathscr{D}(\mathscr{A})=\mathscr{D}(A) \times V
$$

is the infinitesimal generator of the strongly continuous semigroup $\mathscr{L}(t) \in \mathscr{L}(\mathscr{H})$ which is given by

$$
\mathscr{L}(t) x=\left[\begin{array}{l}
\sum_{n=1}^{\infty}\left[\left(\cos \omega_{n} t\right)\left\langle x_{0}, \phi_{n}\right\rangle+\omega_{n}^{-1}\left(\sin \omega_{n} t\right)\left\langle x_{1}, \phi_{n}\right\rangle\right] \phi_{n} \\
\sum_{n=1}^{\infty}\left[\omega_{n}\left(\sin \omega_{n} t\right)\left\langle x_{0}, \phi_{n}\right\rangle+\left(\cos \omega_{n} t\right)\left\langle x_{1}, \phi_{n}\right\rangle\right] \phi_{n}
\end{array}\right] .
$$

Finally, we introduce the spaces

$$
\begin{gathered}
\mathscr{W}=\left\{x \in \mathscr{H} \mid \sum_{n=1}^{\infty} \gamma_{n}\left[\left|\lambda_{n}\right|\left\langle x_{0}, \phi_{n}\right\rangle^{2}+\left\langle x_{1}, \phi_{n}\right\rangle^{2}\right]<\infty\right\}, \\
\mathscr{V}^{*}=\left\{x \in \mathscr{H} \mid \sum_{n=1}^{\infty} \beta_{n}^{-1}\left[\left|\lambda_{n}\right|\left\langle x_{0}, \phi_{n}\right\rangle^{2}+\left\langle x_{1}, \phi_{n}\right\rangle^{2}\right]<\infty\right\}
\end{gathered}
$$

and the operators $\mathscr{B} \in \mathscr{L}(U, \mathscr{V}), \mathscr{C} \in \mathscr{L}(\mathscr{W}, Y)$ by

$$
\mathscr{B}=\left[\begin{array}{l}
0 \\
B
\end{array}\right], \quad \mathscr{C}=\left[\begin{array}{ll}
C & 0
\end{array}\right] .
$$

Then (4.22) is equivalent to the Cauchy problem

$$
\dot{x}=\mathscr{A} x+\mathscr{B} u, \quad y=\mathscr{C} x,
$$

by means of the identification $x=(z, \dot{z})$. Note that we identify $\mathscr{H}=V \times H$ with its dual.

LEMMA 4.8. (i) If

$$
\sup _{n \in \mathbb{N}} \gamma_{n}\left\|b_{n}\right\|_{U}^{2}<\infty
$$

then the operator $\mathscr{B}$ satisfies (H1).

(ii) If

$$
\sup _{n \in \mathbb{N}} \frac{\left\|c_{n}\right\|_{Y}^{2}}{\beta_{n}\left|\lambda_{n}\right|}<\infty,
$$

then the operator $\mathscr{C}$ satisfies $(\mathrm{H} 2)$.

Proof. Statement (ii) is the dual of (i). In order to prove statement (i), note first that

$$
\int_{0}^{T} \mathscr{L}(T-s) \mathscr{B} u(s) d s=\left[\begin{array}{l}
\sum_{n=1}^{\infty} \omega_{n}^{-1} \int_{0}^{T}\left(\sin \omega_{n}(T-s)\right)\left\langle b_{n}, u(s)\right\rangle d s \phi_{n} \\
\sum_{n=1}^{\infty} \int_{0}^{T}\left(\cos \omega_{n}(T-s)\right)\left\langle b_{n}, u(s)\right\rangle d s \phi_{n}
\end{array}\right]
$$


for every $u(\cdot) \in L^{2}[0, T ; U]$ and hence

$$
\begin{aligned}
& \left\|\int_{0}^{T} \mathscr{L}(T-s) \mathscr{B} u(s) d s\right\| \\
& =\sum_{n=1}^{\infty} \gamma_{n}\left\{\left[\int_{0}^{T}\left(\sin \omega_{n}(T-s)\right)\left\langle b_{n}, u(s)\right\rangle d s\right]^{2}\right. \\
& \left.\quad+\left[\int_{0}^{T}\left(\cos \omega_{n}(T-s)\right)\left\langle b_{n}, u(s)\right\rangle d s\right]^{2}\right\} \\
& \leqq\left(\sup _{n \in \mathbb{N}} \gamma_{n}\left\|b_{n}\right\|^{2}\right) \sum_{n=1}^{\infty}\left\{\left\|\int_{0}^{T}\left(\sin \omega_{n}(T-s)\right) u(s) d s\right\|^{2}\right. \\
& \left.\quad+\left\|\int_{0}^{T} \cos \omega_{n}(T-s) u(s) d s\right\|^{2}\right\} \\
& \leqq \text { const. }\left(\sup _{n \in \mathbb{N}} \gamma_{n}\left\|b_{n}\right\|^{2}\right) \cdot\|u(\cdot)\|_{L^{2}[0, T ; U] \cdot}^{2}
\end{aligned}
$$

The final inequality is a consequence of (4.23) together with some properties of Fourier series (see Ingham [13] and Russell [24]).

The next lemma shows under which conditions the spaces $\mathscr{W}$ and $\mathscr{V}$ can be chosen in such a way that (H1), (H2), (H3) are satisfied if the sequences $b_{n}, c_{n}$ are given.

LEMMA 4.9. Let the sequences $b_{n} \in U, c_{n} \in Y, \lambda_{n} \in \mathbb{R}$ be given such that (4.23) is satisfied. Then there exist positive sequences $\beta_{n}, \gamma_{n}$ satisfying (4.24), (4.26) and (4.27) if and only if

$$
\sum_{n=1}^{\infty} \frac{\left\|b_{n}\right\|^{2} \cdot\left\|c_{n}\right\|^{2}}{\left|\lambda_{n}\right|}<\infty .
$$

Furthermore, if (4.28) holds, then the sequences $\beta_{n}, \gamma_{n}$ can be chosen such that $\mathscr{D}_{V}(\mathscr{A}) \subset$ $\mathscr{W} \subset \mathcal{V}$.

Proof. The necessity of (4.28) is obvious. Conversely, if (4.28) holds, then it is easy to see that the sequences

$$
\begin{aligned}
& \beta_{n}=\left\{\begin{array}{l}
\left\|c_{n}\right\|^{2} /\left|\lambda_{n}\right|, \quad\left\|b_{n}\right\|_{U}\left\|c_{n}\right\| \geqq 1 \quad \text { or } b_{n}=0, \quad c_{n} \neq 0, \\
1 /\left\|b_{n}\right\|^{2}\left|\lambda_{n}\right|, \quad\left\|b_{n}\right\|\left\|c_{n}\right\| \leqq 1 \quad \text { and } b_{n} \neq 0, \\
1 /\left|\lambda_{n}\right|, \quad b_{n}=0, \quad c_{n}=0,
\end{array}\right. \\
& \gamma_{n}=\left\{\begin{array}{l}
1 /\left\|b_{n}\right\|^{2}, \quad b_{n} \neq 0, \\
\left\|c_{n}\right\|^{2}, \quad b_{n}=0, \quad c_{n} \neq 0, \\
1, \quad b_{n}=0, \quad c_{n}=0,
\end{array}\right.
\end{aligned}
$$

satisfy the requirements of the lemma. In particular $\beta_{n} / \gamma_{n}$ is bounded and $\gamma_{n} \leqq \beta_{n}\left|\lambda_{n}\right|$ for every $n \in \mathbb{N}$.

We are now in the position to apply Theorem 2.7 to the Cauchy problem (4.25) with the performance index

$$
J(u)=\int_{0}^{T}\left[\|y(t)\|^{2}+\|u(t)\|^{2}\right] d t
$$

Hence there exists a unique nonnegative strongly continuous operator $\mathscr{P}(t) \in \mathscr{L}\left(\mathscr{V}, \mathscr{V}^{*}\right)$ satisfying the differential Riccati equation

$$
\begin{aligned}
& \frac{d}{d t} \mathscr{P}(t) x+\mathscr{A}^{*} \mathscr{P}(t) x+\mathscr{P}(t) \mathscr{A} x-\mathscr{P}(t) \mathscr{R} \mathscr{B} * \mathscr{P}(t) x+\mathscr{C}^{*} \mathscr{C} x=0, \\
& \mathscr{P}(T) x=0, \quad x \in \mathscr{D}_{\mathscr{V}}(\mathscr{A}) .
\end{aligned}
$$


Furthermore, the optimal control is given by the feedback law

$$
u(t)=-\mathscr{B} * \mathscr{P}(t) x(t) .
$$

Example 4.10. As a specific example we consider the system

$$
\begin{array}{ll}
z_{t t}=z_{\xi \xi}, \quad 0<\xi<1, \quad t>0, & \\
z(t, 0)=u(t), \quad z(t, 1)=0, & t>0, \\
y(t)=\int_{0}^{1} c(\xi) z(t, \xi) d \xi, & t>0,
\end{array}
$$

in the Hilbert space

$$
\mathscr{H}=H_{0}^{1}[0,1] \times L^{2}[0,1]
$$

which we identify with its dual. Then the operator $A=\Delta: H^{2}[0,1] \cap H_{0}^{1}[0,1] \rightarrow L^{2}[0,1]$ has the eigenvalues $\lambda_{n}=n^{2} \pi^{2}$ with corresponding eigenfunctions $\phi_{n}(\xi)=\sqrt{2} \sin n \pi \xi$. Furthermore, the input operator for (2.32) takes the form $B u=-\delta^{\prime} u$, where $\delta^{\prime}$ is the distributional derivative of the Dirac delta impulse at $\xi=0$ (see Curtain and Pritchard [4]). Hence

$$
b_{n}=\sqrt{2} n \pi, \quad c_{n}=\sqrt{2} \int_{0}^{1} c(\xi) \sin n \pi \xi d \xi
$$

for $n \in \mathbb{N}$. So condition (4.28) is satisfied if $c_{n}$ is square integrable. The proof of Lemma 4.9 shows that we may choose

$$
\beta_{n}=\max \left\{\frac{\left|c_{n}\right|^{2}}{\left|\lambda_{n}\right|}, \frac{1}{\left|\lambda_{n}\right|^{2}}\right\}, \quad \gamma_{n}=\frac{1}{\left|\lambda_{n}\right|}, \quad n \in \mathbb{N} .
$$

In particular this means that the boundary control system (4.32) has continuous solutions in the space

$$
L^{2}[0,1] \times H^{-1}[0,1]
$$

for every input $u(\cdot) \in L^{2}[0, T]$. This result has also been established by Lasiecka and Triggiani [17]. For the output operator we can allow an arbitrary bounded linear map from $L^{2}[0,1]$ into $\mathbb{R}$. The space $\mathscr{V}$ depends on this map. In any case $\mathscr{W} \subset \mathscr{V}$ and hence $\mathscr{P}(t): \mathscr{V} \rightarrow \mathscr{V}^{*}$ has a smoothing effect with respect to $\mathscr{W}$.

Remark 4.11. An analogous result has been developed by Lasiecka and Triggiani [18] for the higher dimensional wave equation. In their paper the output operator is the identity on the displacement component of the state in $W=L^{2} \times H^{-1}$. This case cannot be treated within our framework. However, the results in [18] are weaker than ours. The uniqueness for the solution of the Riccati equation has not been established in [18]. Furthermore, the Riccati operator in [18] is in $\mathscr{L}(\mathscr{W})$ and does not have smoothing properties with respect to $W$. Consequently the feedback operator becomes unbounded with respect to this space. It seems that for hyperbolic PDE's our assumptions are close to the weakest possible in order to derive a bounded feedback operator.

\section{REFERENCES}

[1] A. V. BALAKRISHNAN, Boundary control of parabolic equations: $L-Q-R$ theory, in Theory of Nonlinear Operators, Proc. 5th International Summer School, Akademie-Verlag, Berlin, 1978.

[2] A. Bensoussan, M. C. Delfour AND S. K. Mitter, The linear quadratic optimal control problem for infinite dimensional systems over an infinite horizon: survey and examples, 1976 IEEE Conference on Decision and Control, IEEE Publications, New York, 1976, pp. 745-751. 
[3] J. A. BURNS, T. L. HeRdMAN AND H. W. STECH, Linear functional differential equations as semigroups in product spaces, SIAM J. Math. Anal., 14 (1983), pp. 98-116.

[4] R. F. CURTAIn ANd A. J. Pritchard, Infinite Dimensional Linear Systems Theory, Lecture Notes in Computer and Information Science 8, Springer-Verlag, Berlin, 1978.

[5] R. DATKO, Uniform asymptotic stability of evolutionary processes in Banach space, SIAM J. Math. Anal., 3 (1972), pp. 428-454.

[6] - Unconstrained control problems with quadratic cost, this Journal, 11 (1973), pp. 32-52.

[7] - Neutral autonomous functional equations with quadratic cost, this Journal, 12 (1974), pp. 70-82.

[8] M. C. Delfour, The linear quadratic optimal control problem with delays in the state and control variables: A state space approach, Centre de Recherche de Mathématiques Appliquées, Université de Montréal, CRMA-1012, 1981.

[9] F. FLANDOLI, Riccati equation arising in a boundary control problem with distributed parameters, this Journal, 22 (1984), pp. 76-86.

[10] J. S. GiBSON, The Riccati integral equations for optimal control problems on Hilbert space, this Journal, 17 (1979), pp. 537-565.

[11] D. HENRY, Linear autonomous neutral functional differential equations, J. Differential Equations, 15 (1974), pp. 106-128.

[12] A. ICHIKAWA, Quadratic control of evolution equations with delays in control, this Journal, 20 (1982), pp. 645-668.

[13] A. E. INGHAM, Some trigonometrical inequalities on the theory of series, Math. Z., 41 (1936), pp. 367-379.

[14] K. ITO AND T. J. TARN, A linear quadratic control problem for neutral systems, J. Nonlinear Anal.-TMA, to appear.

[15] T. Kato, Perturbation Theory of Linear Operators, Springer-Verlag, New York, 1966.

[16] I. LASIECKA AND R. TRIGGIANI, Dirichlet boundary control problem for parabolic equations with quadratic cost: analyticity and Riccati feedback synthesis, this Journal, 21 (1983), pp. 41-67.

[17] - Riccati equations for hyperbolic partial differential equations with $L_{2}\left(0, T ; L_{2}(\Gamma)\right)$-Dirichlet boundary terms, this Journal, 24 (1986), pp. 884-925.

[18] - An $L^{2}$ theory for the quadratic optimal cost problem of hyperbolic equations with control in the Dirichlet boundary conditions, in Control Theory for Distributed Parameter Systems and Applications, F. Kappel, K. Kunisch and W. Schappacher, eds., Lecture Notes in Computer and Information Science 54, Springer-Verlag, Berlin, 1983, pp. 138-152.

[19] J. L. LIONS, Optimal Control of Systems Governed by Partial Differential Equations, Springer-Verlag, New York, 1971.

[20] D. L. LukES AND D. L. Russell, The quadratic criterion for distributed systems, this Journal, 7 (1969), pp. 101-121.

[21] L. PANDOLFI, Stabilization of neutral functional differential equations, J. Opt. Theory Appl., 20 (1976), pp. 191-204.

[22] J. Pollack AND A. J. Pritchard, The infinite time quadratic cost control problem for distributed systems with unbounded control action, J. Inst. Math. Appl., 25 (1980), pp. 287-309.

[23] D. L. RUSSELL, Quadratic performance criteria in boundary control of linear symmetric hyperbolic systems, this Journal, 11 (1973), pp. 475-509.

[24] —_, Closed loop eigenvalue specification for infinite dimensional systems: augmented and deficient hyperbolic cases, MRC, University of Wisconsin-Madison, TSR \#2021, 1979.

[25] D. SAlamon, Control and Observation of Neutral Systems, RNM 91, Pitman, London, 1984.

[26] M. SORINE, Une resultat d'existence et unicité pour l'équation de Riccati stationnaire, Rapport INRIA, no. $55,1981$.

[27] — Sur le semegroupe non linéaire associé a l'équation de Riccati, Centre de Recherche de Mathématiques Appliquées, Université de Montréal, CRMA-1055, 1981.

[28] J. C. WILLEMS, Least squares stationary optimal control and the algebraic Riccati equation, IEEE Trans. Automat. Control, AC-16 (1972), pp. 621-634.

[29] W. M. Wonham, Linear Multivariable Control: A Geometric Approach, Lecture Notes in Economics and Mathematical Systems 101, Springer-Verlag, New York, 1974.

[30] J. ZABCZYK, Remarks on the algebraic Riccati equation in Hilbert space, Appl. Math. Opt., 2 (1976), pp. 251-258. 